\title{
ВОЛНН ПРОСВЕТЛЕНИЯ В ОПТИЧЕСКИ ПЛОТНЫХ СРЕДАХ
}

\author{
В. Е. Харциев \\ Физихо-техничесхий институп, им. А. Ф. Иоффе АН СCCP, \\ Ленинград, СССР \\ (Получили 1 Сентлбрь 1966)
}

\begin{abstract}
Аннотатция
Рассматриватоля явления, имеючие меспо при прохохдении импульсов монохроматического излучения через оптичесои плотнуло на частоте перехода $v_{21}$ среду, содерхашую иентры резонансно поглощалиие излучение. При этом под действием импульса резонансного иялученил происходит насыщение поглощения в слое среды, в результате чего поглощение в нем падает и иялучение проникает в следупоиий слой. Таким образом, просветленкал область непрерывно перемещаетсл в илубь среды, проиесс проветления имеет волноподобньй характер, а плотность фотонов и коэфббииент поглощения в глубине среды при постолнной плотности потока падающих фотонов и однородном распределении поглощающи иентров завислм полько от аргумента $t-x / v$, причем скорость волны просветления $v$ мохет бымь на несколько порлоков менъще скорости света. Механизм насьщения поглощения мохет бымь различным. Необходимым условием для возникновения волн просветления являетсл малая величина потерь фотонов в просветленной части среды. Условия осуществления молн просветления реалияуются для многих хонденсированных сред, обладахощих линиями интенсивного поглощения, и особенно благоприяпны при наличии мехду уровнями 1 и 2 метастабильных уровней (системы типа рубина). Эфбект волн просветления являетсл общим для различных спектральных областей электромагнитного иялученил и мохет реалияоваться в астрофияических масштабах.
\end{abstract}

\section{Введение}

ри взаимодействии потоков мощного монохроматического излучения с конденсированной средой могут меть место процессы резонансного поглощения [1], отражения и рассеяния без изменения частоты, елинейные оптические явления типа генерации гармоник $[2,3]$, рассеяние Бриллюэна - Мандельштама $4,5]$ индуцированное косбинационное рассеяние [6]. В случае сильно поглощающих сред при наличии езонансного поглощения этот механизм до насыщенияя поглощения может быть доминирующим. Эффект асыщения поглощения, подробно исследовавшийся для случая слабо поглощаюихся сред [9 - 15], в лучае сильно поглощаюих сред характеризуется резкой нестационарностыю и пространственной неднородностью $[7-9]$.

Ниже дается общий анализ явления волн просветления, возникающих в оптически плотной среде од действием импульса мощного монохроматического излучения в случае, когда среда содержит резоансно поголщающие центры одного типа. Другими механизмами взаимодействия излучения со средой ренебрегаем. Аналогичное явление при определенных условиях может иметь место также при других Іеханизмах насьщения поглощения, например, при двухфотонных переходах, фотоионизации атомов и оотодиссоциации молекул в газе. 


\section{1. Качественная картина явления волн просветления}

Пусть поглощающие центры среды имеют систему энергетических уровней, показанную на рис.1. Буд предполагать, что линия перехода 1-2 однородно уширена, а для спектральных ширин импульса $\Delta v_{i}$ и перехода $\Delta v_{21}$ имеет место неравенство $\Delta v_{21} \gg \Delta v_{i}$. Пренебрежем процессами нерезонансного погощения излучения и вкладом переходов $2 \rightarrow 1$ в кинетику просветления среды. Будем считать, длительность импульса $T_{i}$ ограничена условием $\tau_{31} \gg T_{i}$, где $T_{31}$-наименьшее из времен жизни дл переходов типа $3 \rightarrow 1$. Для простоты для статистических весов $f_{\alpha}$ принимаем $f_{1}=f_{2}$.

В принятых приближениях взаимодействие излучения с поглощающим центрами моӝно характеризо вать усредненным по спектральному интервалу $\Delta v_{i}$ параметром $\sigma$-сечением захвата фотона резонанс ного излучения поглощающим центром. Для максимального значения коэффициента поглощения $k_{m}=\sigma /$ оптически плотной среды на частоте $v_{21}$ имеет место соотношение $k_{m} l \gg 1$, где $N$ - плотность по лощающих центров, l-толщина одномерной среды. Среда бхдет прозрачной для резонансного излучен частоты $v_{21}$, если $k=\sigma\left(n_{1}-n_{2}\right) \simeq 0$, где $n_{1}$ и $n_{2}-$ населенности уровней 1 и 2 . Это усло может быть реализовано в двух случаях: (А) $n_{1} \simeq n_{2} \simeq 0$ : (Б) $n_{1} \simeq n_{2} \neq 0$. Рассмотрим качественно явление просветления оптически плотной среды мощным резонансным излхчением в этих лвух случаях.

Случай А соответствует трехуровневой системе, в которой уровепь 2 играет роль промежуточноп уровня. При этом в слое одномерной среды толщиной $\Delta l \simeq 1 / k_{m}$ под возлействием резонансного излхчения имеет место тенденция выравнивания населенностей уровней 1 и 2 , но из-за переходов канале $2 \rightarrow 3 n_{1}$ и $n_{2}$ не могут сравняться. В результате этого населенность уровня 1 уменьшается населенность уровня 3 увеличивается, коэффициент поглощения резонансного излучения в слое уменьшается, и излучение проникает в следующий слой. Таким образом, под воздействием импульса резонансного излучения просветленная область непрерывно перемещается в соеденить глубь среды, т.е. имеет место волноподобный процесс. В конечном состоянии все поглощающе центры переходят состояние, жоответствующее уровням типа 3 , и среда становится прозрачной для резонансного излу чения частоты $v_{21}$. Скорость перемещения просветляющейся области - волны просветления - при мал плотностях потока фотонов в пренебрежении релаксацией в канале $2 \rightarrow 1$ тожно оценить следующим образом. Для просветления объема $S \Delta l$ одномерной среды с поперечным сечением $S$ необходимо возбудить $N S \Delta l$ поглщщающих центров. Для этого необходим поток фотонов $u_{0} C S$ длительности $\Delta t$, где $u_{0}$ - начальная плотность фотонов, с-скорость света в среде. В результате для скорости волны просветления получаем

Случай Б соответствует изолированной двухуровневой системе. Фронт волны просвеления предста ляет собой область насьщения поглощения в канале 1 - 2. Для скорости перемещения волны просвет ления по аналогии с предыдущим случаем получаем

\section{2. Исходные уравнения}

При использованных предположениях кинетика распространения импульса резонансного излучения частоты $v_{21}$ в одномерной среде с энергетическими уровнями поглощающих центров, показанньми на 
рис.1, описывается системой уравнений

$$
\begin{aligned}
& \frac{\partial u}{\partial t}+c \frac{\partial u}{\partial x}=-\sigma c\left(n_{1}-n_{2}\right) u \\
& \frac{\partial n_{1}}{\partial t}=-\sigma c\left(n_{1}-n_{2}\right) \dot{u}+\frac{n_{2}}{\tau_{21}} \\
& \frac{\partial n_{2}}{\partial t}=\sigma c\left(n_{1}-n_{2}\right) u-\left(\frac{1}{\tau_{21}}+\frac{1}{\tau_{23}}\right) n_{2} \\
& n_{1}+n_{2}+n_{3}=N
\end{aligned}
$$

с начальными и граничными условиями для плотности фотонов в среде $u$ и населенностей энергетических уровней $n_{i}$

$$
a(x=0, t)=u_{0}(t), \quad n_{1}(x, t=0)=N(x), \quad n_{2,3}(x, t=0)=0 .
$$

Для двухуровневой системы (случай Б) процесс просветления описывается системой уравнений $(2.1)$ и $(2.6)$

$$
\frac{\partial n}{\partial t}=-2 \sigma c n u
$$

причем $n=n_{1}-n_{2}$. Волноподобное просветление среды в этом случае может возникнуть при выполнении условия $\tau_{2} \gg T_{i}$, где $\tau_{2}=\left(\frac{1}{\tau_{21}}+\frac{1}{\tau_{23}}\right)^{-1}$, Время просветления $T=l / v$. В случае А эффективное просветление среды может иметь место, если в процессе просветления выполняется условие $T \gg \tau_{23}$. В промежуточном случае $T_{i} \simeq \tau_{21} \simeq \tau_{23}$ волна просветления ни в двухуровневом, ни в трехуровневом варианте не успевает сформироваться.

Рассмотрим случай волны просветления для трехуровневых систем (А) при достаточно малом времени $\tau_{23}$, когда для уровня 2 имеет место условие квазистационарности $\partial n_{2} / \partial t<\sigma$ спи. Тогда вычитая из уравнения (2.2) уравнение (2.3) и пользуясь условием квазистационарности, получаем

$$
\frac{\partial n}{\partial t}=-r \sigma c u n
$$

где

$$
r=\frac{T_{21}}{T_{23}+T_{21}}
$$

Таким образом, в случае А при квазистационарном приближении просветление среды описывается системой уравнений $(2.1)$ и (2.7), совпадающей с точностыо до множителя $r$ с системой уравнений для случая Б. 


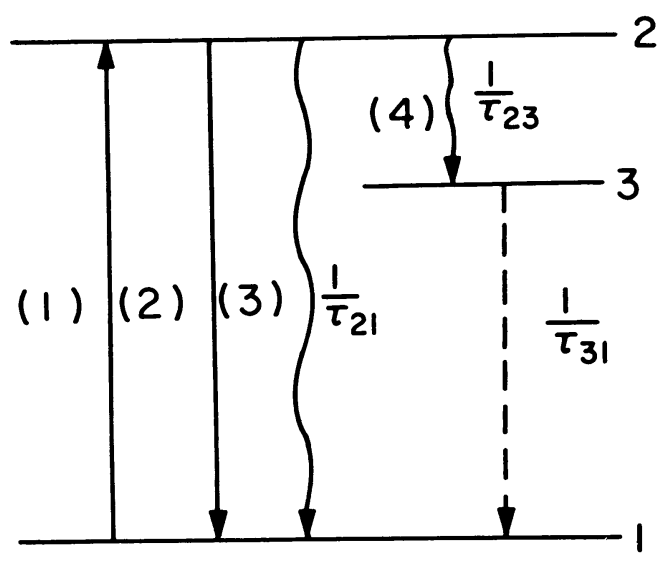

Рис. 1.

Схема энергетических уровней и переходов в поглощаюих центрах. Непрерывными линиями показаны переходы, учтенные в системе уравнений (2.1) - (2.3):

1 - поглощение, 2 - индуцированное излучение, 3 и 4 - распад возбужденного состояния $2 ; \tau_{i k}^{-1}-$ вероятность переходов в канале $i \rightarrow k$.

Следует также отметить, что область применимлсти уравнений $(2.1)-(2.3)$ ограничена требованиє медленности изменения величин $u$ и $n_{i}$ за время поперечной релаксации перехода 2-1 $T_{21}$, т.е. услф виями $\Delta t \gg T_{21}, \Delta x \gg c T_{21}, \quad n \gg c T_{21} \frac{\partial n}{\partial x}$ и т.д. Поскольку ширина фронта волны просветления по порядку величины равна $1 / k_{m}$, то для применимости уравнений $(2.1)-(2.3)$ на этих расстояниях необходимо выполнение условия $1 / k_{m} \gg c T_{21}$, из-которого следует критерий применимост этих уравнений $\sigma \mathrm{NcT} T_{21} \ll 1$. Ввиду малости $T_{21}\left(T_{21} \simeq 10^{-12}\right.$ сек) этот критерий обычно выполняется даже для сред с большими коэффициентами поглощения.*

\section{3. Стационарные волны просветления}

Рассмотрим процесс просветления среды в случае А при импульсе излучения постоянной интенсивности и однородном распределении поглощающих центров в среде. В глубине просветленной среды в принятых приблихениях потерь фотонов частоты $v_{21}$ не происходит. Поглощение имеет место на фронт волны просветления, где происходит перекачка поглощаюих центров из состояний 1 в состояния 3 . В этих условиях в среде устанавливается стационарный фронт волны просветления. Тогда нелинейная

* Наличие резонансного монохроматического излучения должно приводить к измененио формы линии. в зависимости от плотности потока излучения [17]. Этот эффект мы не учитываем. 
истема уравнений в частных производных $(2.1)-(2.3)$ имеет решения, зависящие только от аргумента $=t-x / v$ и может быть сведена к системе обыкновенных дифференциальных уравнений. При этом равнение (2.1) запишется в виде

$$
u_{\theta}^{1}=q \sigma c n u
$$

де $q=\left(\frac{c}{v}-1\right)^{-1}$, а в уравнениях $(2.2)$ и (2.3) дифференцирование по $t$ заменится на диффеенцирование по $\theta$. Новой системе уравнений соответствуют некоторые "начальные" условия на ронте волны $\theta_{0}$

$$
u\left(\theta=\theta_{0}\right)=u^{(0)} \quad n_{i}\left(\theta=\theta_{0}\right)=n_{i}^{(0)}
$$

I условия асимптотического типа

$$
u(\theta \rightarrow \infty) \rightarrow u_{0}, \quad n_{1,2}(\theta \rightarrow \infty) \rightarrow 0, \quad n_{3}(\theta \rightarrow \infty) \rightarrow N .
$$

Получим первый интеграл системы уравнений для стационарных волн просветления без предпологения о квазистационарности для уровня 2. Преобразуя уравнения для населенностей уровней, полуเаем соотношение для производных по переменной $\theta$, соответствующее условию стационарности проlecca

$$
u_{\theta}^{1}=\frac{q}{2}\left[\left(1+2 \frac{T_{23}}{T_{21}}\right)\left(n_{3}\right)_{\theta}^{1}-n_{\theta}^{1}\right] .
$$

Іосле интегрирования (3.4) с учетом условий (3.2) и (3.3) получаем общее соотношение, бвязызающее параметры на фронте стационарной волны просветления

$$
u_{0}-u^{(0)}=\frac{q}{2}\left[\left(1+2 \frac{T_{23}}{\tau_{21}}\right)\left(N-n_{3}^{(0)}\right)+n^{(0)}\right] \text {. }
$$

Іспользование соотношения (3.4) приводит к системе двух нелинейных уравнений, которые могут Быть интегрированы численно.

В квазистационарном приближении уравнение (3.1) должно быть дополнено уравнением

$$
n_{\theta}^{1}=-r \sigma c n u
$$

з уравнений (3.1) и (3.6) с учетом (3.2) получаем

$$
u-u^{(0)}=(q / r)\left(n^{(0)}-n\right)
$$

иричем $n^{(0)}$ определяется при учете асимптотического условия (3.3) $n^{(0)}=(r / q)\left(u_{0}-u^{(0)}\right)$. Гаким образом, плотность фотонов на фронте волны определяется уравнением

$$
u_{\theta}^{1}=r \sigma c\left(u_{0}-u\right) u \text {. }
$$


Решение уравнения (3.7) дает вырахения для стационарных волн просветления

$$
\begin{aligned}
& u(\theta)=u_{0}\left\{1+\left(\frac{u_{0}}{u(0)}-1\right) \exp \left[-\left(\theta-\theta_{0}\right) r \sigma c u_{0}\right]\right\}^{-1} \mid \\
& k(\theta)=\frac{\sigma u_{0} r}{q}\left\{1+\left(\frac{u_{0}}{u(0)}-1\right) \exp \left[\left(\theta-\theta_{0}\right) r \sigma c u_{0}\right]\right\}^{-1} \\
& n_{2}(\theta)=r \sigma \operatorname{cun} \tau_{23}=\frac{r^{2} \sigma c \tau_{23} u_{0}^{2}}{4 q}\left\{\operatorname{Ch}\left[\frac{1}{2}\left(\theta-\theta_{0}\right) r \sigma c u_{0}-\ln \left(\frac{u_{0}}{u^{(0)}}-1\right)\right]\right\}^{-2}
\end{aligned}
$$

В соотношениях (3.8)-(3.10) величины $u^{(0)}$ и $v$ предполагаются заданными. При $r=2$ соотнотения (3.8) и (3.9) описывают процесс просветления для двухуровневых систем (случай Б).

\section{4. Общие решения}

Рассмотрим теперь решение уравнений (2.1) и (2.7) с условиями (2.5) в общем случае, когда плотность потока монохроматического излучения зависит от времени, а распределение резонансно поглощающи центров в среде является неоднородным. Получаемые ниже решения являются точными в лвух случаях: (1) случай А - система трех энергетических уровней при условии квазистационарнос уровня $2 ;(2)$ - случай Б $(r=2)$ - изолированная двухуровневая система.

Для интегрирования системы $(2.1),(2.7)$ воспользуемся одним из вариантов метода Дородницьна [18] (см. также [7]). В этом варианте оказывается возможным свести решение нелинейной системы дифференциальных уравнений в частных производных дивергентного типа к решению обыкновенного дифференциального уравнения, решение которого в свою очередь сводится к квадратурам. Интегрируя уравнение (2.7), получаем

$$
n(x, t)=N(x) \exp \left[-r \sigma c \int_{0}^{t} u\left(x, t_{1}\right) d t_{1}\right]
$$

Подставим формальное решение (4.1) в уравнение (2.1) и, проведя интегрирование по $t$ с учетом равенства $u(x, t=0)=0$, получим

$$
\frac{\partial w}{\partial t}+c \frac{\partial w}{\partial x}=-[1-\exp (-r \sigma c w)] \frac{N(x)}{r}
$$

где $w(x, t)=\int_{0}^{t} u\left(x, t_{1}\right) d t_{1}$. Уравнению (4.2) соответствует граничное условие $w(x=0, t)=$ $\int_{0}^{t} u_{0}\left(t_{1}\right) d t_{1}$. Интегрирование уравнения (4.2) методом характеристик приводит к уравнения 


$$
w_{x}^{1}=-[1-\exp (-r \sigma c w)] \frac{N(x)}{r}
$$

с условием $w(x=0)=\int_{0}^{\psi} u_{0}\left(t_{1}\right) d t_{1}$, где $\psi=t-x / c$. Решение уравнения (4.3) имеет вид

$$
w(x, t)=(r \sigma c)-1 \ln \left[1+\frac{A\left(t-\frac{x}{c}\right)-1}{B(x)}\right]
$$

где

$$
A\left(t-\frac{x}{c}\right)=\exp \left(r \sigma c \int_{0}^{t-\frac{x}{c}} u_{0}\left(t_{1}\right) d t_{1}\right), \quad B(x)=\exp \left(\sigma \int_{0}^{x} N\left(x_{1}\right) d x_{1}\right)
$$

Учитывая определение для величины $w(x, t)$ и соотношение $(4.1)$, получаем общие решения системы $(2.1),(2.7)$ :

$$
\begin{aligned}
& u(x, t)=u_{0}\left(t-\frac{x}{c}\right)\left[1+\frac{B(x)}{A\left(t-\frac{x}{c}\right)}-A^{-1}\left(t-\frac{x}{c}\right) \cdot\right]^{-1} \\
& n(x, t)=N(x)\left[1+\frac{A\left(t-\frac{x}{c}\right)}{B(x)}-B^{-1}(x)\right]^{-1}
\end{aligned}
$$

Решения $(4.6),(4.7)$ имеют в глубине среды (при $A \gg 1, B \gg$ ) волноподобный характер, при этом $\frac{n}{N}=\frac{u}{u_{0}} \frac{A}{B}$. В случае однородного распределения поглощающх центров скорости перемещения состояний среды с равными коэффицентами поглощения $v^{(k)}$ и равными плотностями фотонов $v^{(u)}$, определяемые из условий $d n / d t=0$ и $d u / d t=0$, имеют вид

$$
v(k)=\frac{r u_{0} c}{N+r u_{0}} \quad v(u)=\frac{r u_{0}+m}{N+r u_{0}+m} c
$$

где $m=\left(\sigma c u_{0}\right)^{-1}\left(\frac{A}{B}+1\right) \frac{\partial u_{0}}{\partial t}$. Таким образом, в общем случае $v(k) \neq v(u)$. Для случая $u_{0}=$ const. $N=$ const. $v^{(k)}=v^{(u)}$. При этом из соотношений (4.6) и (4.7) получаем решения, имеюще в глубине оптически плотной среды на частоте $v_{21}$ явную волноподобную форму

$$
\begin{aligned}
& u(x, t)=u_{0}\left\{1+\exp \left[-\left(t-\frac{x}{v(k)}\right) r \sigma c u_{0}\right]-\exp \left[-\left(t-\frac{x}{c}\right) r \sigma c u_{0}\right]\right\}^{-1} \\
& n(x, t)=N\left\{1+\exp \left[\left(t-\frac{x}{v(k)}\right) r \sigma c u_{0}\right]-\exp [-\sigma N x]\right\}^{-1}
\end{aligned}
$$




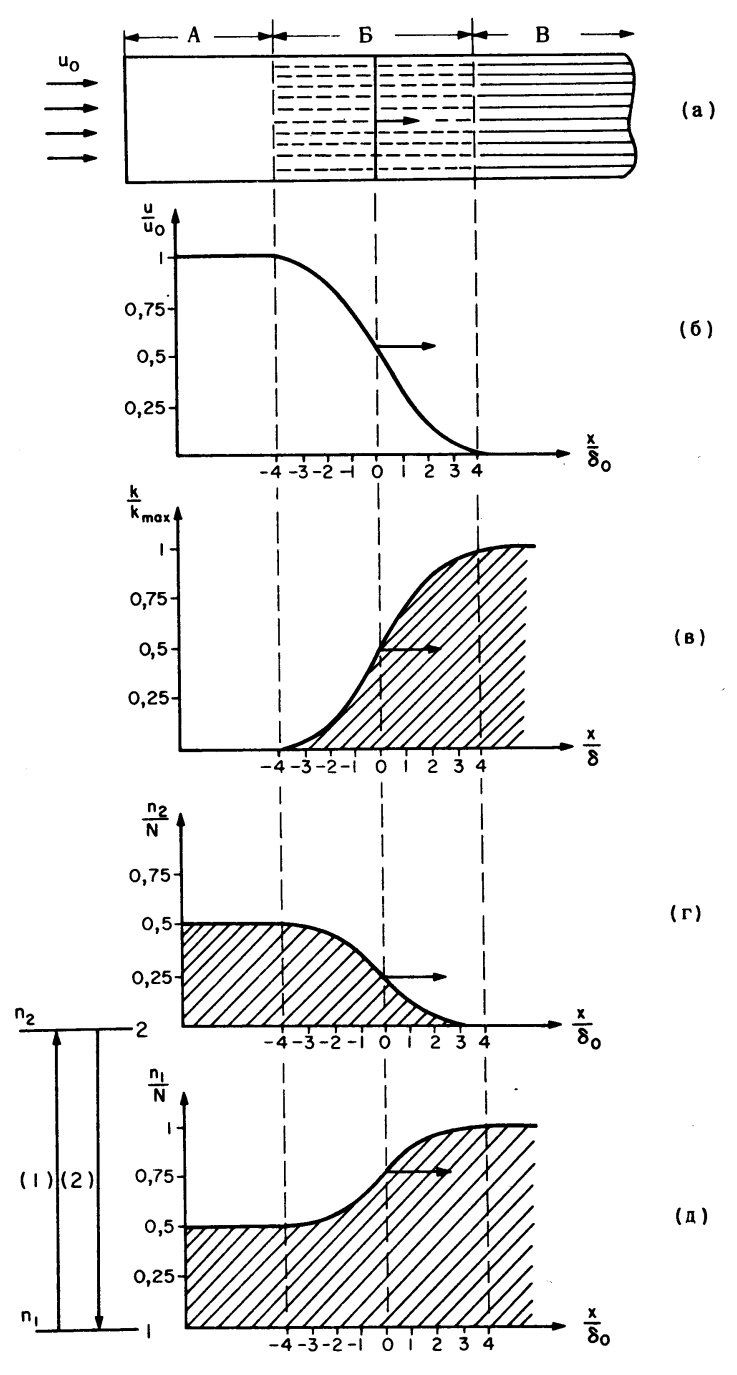

Рис. 2.

Профили волны просветления в двухуровневых системах в случае однородного распределения поглощаюих центров и импульса постоянной интенсивности при учете процессов поглощения (1) и индуцированного излучения (2): а) просветленная область (А), просветляющийся слой (Б) и непросветленная область (В) оптически плотной среды; изменение плотности потока фотонов (б), коэффициента поглощения (в) и населенностей уровней 2 (г) и 1 (д) соответственно. Начало отсчета безразмерного переменного $x / \delta_{0}$ совмещено с точкой, для которой $u / u_{0}=k / k_{m}=1 / 2$. Направление движения волны указано стрелками при $x / \delta_{0}=\cdot 0$.

конечную ширину, определяемую отношением $B / A$ и при вхождении импульса монохроматического излуче ния в среду формируется на конечном расстоянии от ее границы.

Для выполнения условия квазистационарного приближения для уровня 2 необходимо потребовать, чтобы время, в течение которого волна просветления, соответствующая случаю выравнивания 
$n_{2}(x, t)=r \sigma c \tau_{23} u_{0} N\left\{2 \mathrm{Ch}\left[\frac{1}{2}\left(t-\frac{x}{v^{(k)}}\right) r \sigma c u_{0}\right]-C\right\}^{-2}$

где $C=\exp \left\{-\frac{\sigma}{2}\left[N x+\left(t-\frac{x}{c}\right) r c u_{0}\right]\right\}$. При $N \gg r u_{0}$ выражение для $v^{(k)}$ переходит в

случае А при $\tau_{21} \gg \tau_{23}$ в оценочное соотношение $(1.1)$, в случае Б при $r=2$ - в соотношение (1.2). При $r u_{0} \gg N$ просветление распространяется со скоростыю света в среде: $v^{(k)}=v^{(u)}=c$. Соотношения (4.9)-(4.11) согласуются с выражениями (3.8)-(3.10) для стационарных волн просветления в среде (см. также [7-9]). При этом оказывается возможным определить входящие в (3.8)(3.10) неизвестные параметры - скорость волны просветления и значение плотности фотонов на фонте фронте волны просветления: $v=v^{(k)}, u^{(0)}=1 / 2 u_{0}$.

Фронт стационарной волны просветления определяется характерными параметрами среды $k_{m}$, $\tau_{0}=1 / k_{m} c$ и безразмерными отношениями $r, \tau_{23} / \tau_{0}, g=u_{0} / N$. В соответствии с выражениями (4.9) и (4.10) плотность числа фотонов в среде и коэффициент поглощения в случае стационарной волны зависят только от безразмерного переменного $\xi=\frac{x}{\delta_{0}}-\frac{t}{\tau_{0}} g r$, где $\delta_{0}=$ $\left[(1+g r) k_{m}\right]^{-1}$. Полагая $x=0$, совместим начало отсчета времени с моментом, которому соответствуют условия $\frac{u}{u_{0}}=\frac{k}{k_{m}}=\frac{1}{2}$. Тогда зависимость величин $u, k$ и $n_{i}$ от переменной $\xi ;$ в глубине среды определит профили волны просветления в системе координат, связанной с волной $(t=0)$. На рис.2 построен профиль фронта волны просветления для двухуровневой системы (случай Б). Профили населенностей уровней для волны просветления в случае А показаны на рис.3. В этом случае плотность фотонов в среде и коэффициент поглощения в квазистационарном приближении для уровня 2 имеют профили, совпадающие со случаем Б (рис. 2б, 2в), при этом меняется, ввиду зависимости $\delta_{0}$ от параметра $r$, только масштаб пространственной координаты.

Из соотношений $(4.6),(4.7)$ и (4.9)-(4.11) следует, что фронт волны просветления имеет конечную ширину, определяемую отношением $B / A$ и при вхождении импульса монохроматического излучения в среду формируется на конечном расстоянии от ее границы.

Для выполнения условия квазистационарного приближения для уровня 2 необходимо потребовать, чтобы время, в течение которого волна просветления, соответствующая случаю выравнивания заселенностей (Б), проходит расстояние $\Delta$, на котором происходит практически полное поглощение фотонов, значительно превышало время $\tau_{23}$. Для расстояния $\Delta_{p}$, на котором происходит уменьшение плотности фотонов в среде от значения $u_{0}\left(1-10^{-p}\right)$ до $10-p_{u_{0}}$, пользуясь точным (в случае Б) решением (4.9) в гуубине среды, получаем $\Delta_{P}=\frac{2 \ln \left[10^{P}-1\right]}{\sigma\left(N+2 u_{0}\right)}$. Пользуясь выражением (4.8) для $v^{(u)}$ в случае импульса с мало меняющейся во времени интенсивностыю и полагая $p=2$, получаем критерий квазистационарного приближения для уровня 2

$$
\sigma \quad u_{0} c \quad \tau_{23} \ll 5 \text {. }
$$




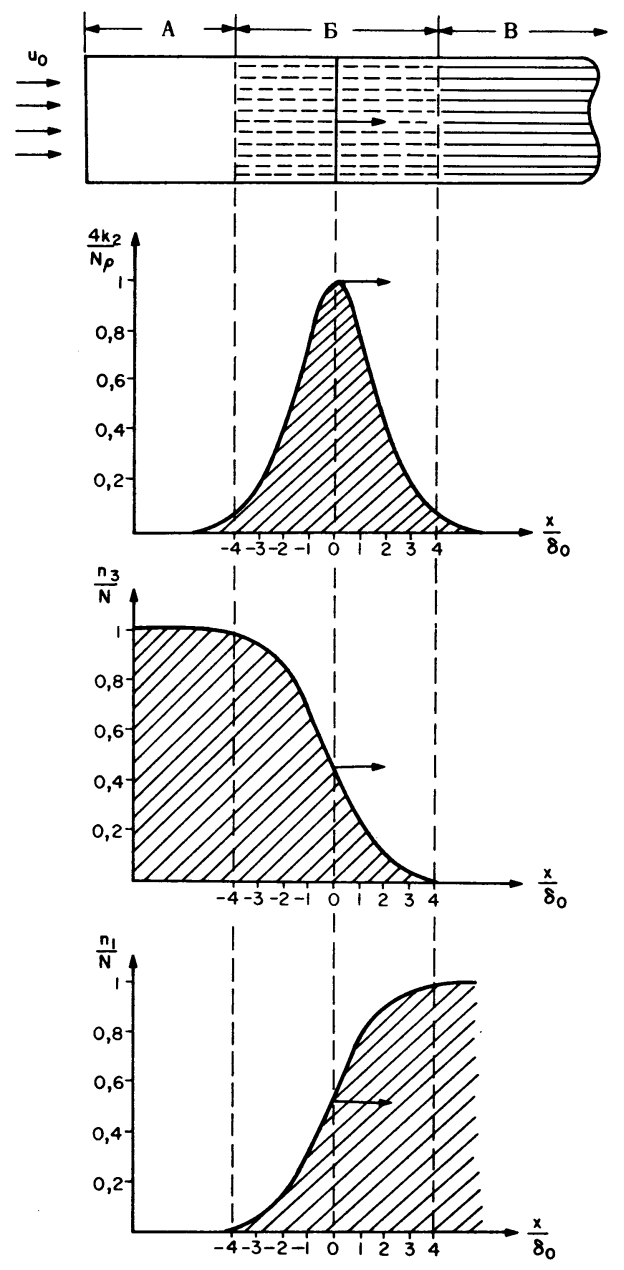

(a)

(6)

(B)

(r)

Puc. 3.

Профили волны просветления в случае трехуровневых систем: (а) три области просветляемой среды; (б) - (г) населенности уровней $n_{2}, n_{3}$ и $n_{1}$, соответственно. Изменение плотности фотонов и коэффициента поглощения $k$ аналогично случало двухровневых систем (рис $2 \sigma$, в); $\rho=r \sigma c u_{0} \tau_{23}=0.1$, при этом $n_{2}$ max $/ N=1 / 40$.

Неравеисво (4.12) можно записать в виде $\tau_{23} \ll 5 / k_{m}$. В дополнение к критерио (4.12) необходимо также потребовать, чтобы выполнялось условие $\tau_{21} \gg \tau_{23}$. При $\tau_{23}=10^{-9}-10^{-8}$ сек критерий (4.12) может быть выполнен для больших плотностей потоков монохроматического излучения. Невыполнение критерия (4.12) приводит к растягиванию фронта волны просветления.

Из соотношения (4.6) при малых плотностях потоков резонансного излучения можно получить известный закон Бугера [19] 


$$
u(x, t)=u_{0}\left(t-\frac{x}{c}\right) \exp \left(-\sigma \int_{0}^{x} N\left(x_{1}\right) d x_{1}\right)
$$

найти условие его применимости в принятых приблихениях

$$
r \sigma c \int_{0}^{t-\frac{x}{c}} u_{0}\left(t_{1}\right) d t_{1} \ll 1 \text {. }
$$

Общее решение (4.6) справедливо при условии, что длительность импульса $T_{i}$ удовлетворяет условиям: в случае А $T_{i} \ll \tau_{31}, T_{21}$, в случае Б $T_{i} \ll \tau_{2}$. Если $u_{0}$ мало меняется за времена $\tau_{31}, \tau_{21}$ и $\tau_{2}$ соответственно и в соотношении $(4.14) t \gg x / c$, то критерии применимости закона Бугера для потоков мощности монохроматического излучения для случаев А и Б можно записать в виде

$$
\begin{aligned}
& P_{A} \ll \frac{h v_{21}}{r \sigma \tau_{31}}, P_{\mathrm{B}} \ll \frac{h v_{21}}{2 \sigma \tau_{2}} \text {. Из последнего неравенства для } R_{1} \text { - линии рубина при } \\
& \sigma=2.5 \times 10^{-20} \mathrm{~cm}^{2} \text { и } \tau_{2}=3 \times 10^{-3} \text { сек получаем } P_{\mathrm{B}} \ll 10^{3} \mathrm{BT} / \mathrm{cm}^{2} .
\end{aligned}
$$

\section{5. Заклочение}

Рассмотренный механизм просветления оптически плотных сред является общим для различных участков электромагнитного спектра. Такой волноподобный процесс, связанный с изменением состояния системы, состоящей из большого числа ғастищ, под действием потока энергии может иметь место при другом виде собственного спектра системы и типов переходов. В качестве примера можно указать на возможный эффект волн просветления под действием двухфотонных переходов в среде, содерхащей поглощающие центры с двумя энергетическими уровнями. Волноподобный процесс просветления может быть доминирующим, если конкурирующие с ним процессы, приводящие к замедленио волны просветления (нерезонансное поглощение и потери излучения на частоте $v_{21}$, связанные с частотным преобразованием излучения), являются малыми. В случае конденсированного состояния к нерезонансным процессам относятся решеточное поглощение, в случае полупроводников (при энергии фотонов меньшеиширины запрещенной зоны и при пренебрехении многофотонными переходами)-поглощение на свободных носителях. Таким образом, волноподобное просветление может иметь место в диэлектриках и полупроводниках в области примесного поглощения, в растворах органических соединений, а такще в газах (в случае, когда столкновения несущественны).

Наибольший интерес представляют условия, в которых время жизни просветленного состояния среды является относительно большим. Для конденсированного состояния такие условия реализуются для случая Б в микроволновом диапазоне, к^гда время жизни определяется спин-решеточной релаксацией и имеющими малую вероятность спонтанными переходами, для случая А - при метастабильности уровня 3. Последний случай может иметь место растворов органических и металлоорганичесоих соединений типа фталоцианинов [20-22]. Поглощащие молекулы в таких растворах обладают сравнительно узкими полосами поглощения в видимой области, большими сечениями захвата фотона резонансного излучения, малыми временами $\tau_{23}$, а уровни 3 являются метастабильными уровнями. Благодаря этим особенностям возможно при сравнительно малой концентращии поглощающи центров создать среду с большой оптической плотносты $\sigma N l$ на частоте $v_{21}$. Для оценки параметров волноподобного просветления таких сред, примем $\sigma=10^{-16} \mathrm{~cm}^{2}, N=10^{17} \mathrm{~cm}^{-3}, l=1 \mathrm{~cm}, c=2 \times 10^{10} \mathrm{~cm} / \mathrm{ceK}$, $T_{23}=3 \times 10^{-9} \mathrm{ceK}, \tau_{21} \gg \tau_{23}, u_{0}=0.5 \times 10^{10} \mathrm{~cm}^{-3}$, что соответствует при $h v_{21}=3 \times 10^{-12}$ эрг плотности потока мощности $P=30 \mathrm{BT} / \mathrm{cm}^{2}$. При этих параметрах $\sigma N l=10$, критерию $(4.12)$ 
соответствует условие $P \ll h \nu_{21} / \sigma_{23}=5 \times 10^{6}$ вт/см ${ }^{2}$. В соответствии с равенством (1.1) скорость волны просветления $v \quad 10 \mathrm{~m} /$ сек, т.е. поток световой энергии в оптически плотной сред может пересещаться со скоростью спринтера на дистанции. При этом время волноподобного просветления жреды $l N / u_{0} c \simeq 10^{-3}$ сек.

В случае газовой среды учет насыщения поглощения, связанный с просветлением среды, особенно существенен для микроволнового диапазона и может значительно изменить величины плотностей потоков энергии на резонансных частотах $[10,16]$. Механизм волноподобного просветления может иметь место и в астрофизических масштабах. Один из возможных эффектов - просветление межзвездной среды под действием потоков излучения переменной интенсивности, возникающих при взрывах оболочек звезд или других нестационарных процессах. При этом время жизни просветленного состояния для микроволновой области спектра определяемое столкновениями между атомами и спонтанным излучением, является макроскопически болышим. В случае распространения волны просветления в среде частично просветленной стационарным потоком излучения скорость волны возрастает.

Пользуемся случаем выразить большую признательность Е.Ф. Гроссу, А.И. Губанову, В.М. 0вчинникову, А.В. Тулубу, Д.А. Варшаловичу, Г.И. Злотнику за обсуждение результатов.

\section{Jитература}

1. A. MItChell и M. ZEMANSKY, Resonance Radiation and Exited Atoms. Cambridge Series, London (1934).

2. С.А. АХМАНОВ и Р.В. ХОХЛОВ, Проблемь нелинейжой опмики. Изд. АН СССР, Москва (1964); А.М. БОНЧ-БРУЕВИЧ и В.А. ХОДОВОЙ, УФН 85, 3 (1965); Л.Н. ОВАНДЕР, УФН 86, 3 (1965).

3. N. BLOEMBERGEN, Nonlinear Optics. Benjamin, New York (1965).

4. R. CHIAO, С. TOWNES и V. STOICHEV, Phys. Rev. Letters 12, 592 (1964); 5, 84 (1964).

5. И.Л. ФАБЕЛИНСКИЙ, Молехулярное рассеяние света. Изд.во "Наука", (1965 г).

6. Е. WOODBURY и พ. NG, Proc. ITE 50, 2367 (1962); В.А. ЗУБОВ, М.М. СУЩИНСКИЙ и И.К. ШУВАЛОВ, УФН 83, 197 (1964).

7. R. Beildmann, G. Birnbaum и W. WAGNeR, J. Appl. Phys. 34, 780 (1963).

8. В.М. ОВЧИННИКОВ и В.Е. ХАРЦИЕВ, ЖЭТФ 49, 315 (1965).

9. В.Е. ХАРІІИЕВ, Оптика и Спектроскопия 20, 514 (1966).

10. В.Л. ГИНЗБУРГ, Изв. АН СССР, сер. физ. 7, 96 (1943); В.Л. ГИНЗБУРГ, УФН 31, 320 (1947).

11. C. TOWNES, Phys, Rev. 70, 665 (1946).

12. R. KARPLUS и J. SCHWINGER, Phys. Rev. 73, 1020 (1948).

13. Н.Г. БАСОВ и А.М. ПРОХОРОВ, УФН 57, 485 (1955).

14. С. TOWNES и A. SHAWLOW, Microwave Spectroscopy. McGraw Hill, New York (1955). 
15. В.М. КОНТОРОВИЧ и А.М. ПРОХОРОВ, ЖЭТФ 33, 1428 (1957); В.М. ФАЙН, УФН 64, 273 (1958); Ф.Н. БУНКИН, Изв. Вузов, Радиофизика 5, 687 (1962).

16. Д.А. ВАРШАЛОВИЧ, Астрономический хурнал 42, 557 (1965).

17. С.Г. РАУТИАН И И.И. СОБЕЛЬМАН, ХЭТФ 41, 456 (1961).

18. Ф.Ф. ДОРОДНИШЫН, Труды Третьеъо Всеслюзного математического съезда 3 , 447. Изд-во АН СССР, Москва (1958ц; R. BELLMANN, G. BIRNBAUM и W. WAGNER, J. Appl. Phys. 34, 780 (1963).

19. Г.С. ЛАНСБЕРГ, Оптика, Гос. ИздБ техн. теор. лим-ры Москва (1957); В.А. ФАБРИКАНТ, Изд. AH CCCP, сер. gиз. 26, 61 (1962).

20. А.Н. ТЕРЕНИН, Сборник Проблемы фотосинтеза, стр. 5. Изд-во АН СССР (1959).

21. Г.П. ГУРИНОВИЧ, А.Н. СЕВЧЕНКО и К.Н. СОЛОВЬЕВ, УФН 79, 173 (1963).

22. R. SOROKIN, J. LUZZI, J. LANKARD и G. PETTIT, I.B.M. J. 8, 182 (1964).

\section{ПРИМЕЧАНИЕ ПІРИ КОРРЕКТУРЕ}

В работе автора [9] отмечалось, что после прохождения волны просветления среда может прийти в инвертированное состояние (т.е. состояние с отрицательной температурой и отрицательным коэффициентом поглощения) по отношению к переходам между некоторыми энергетическими уровнями. Гакое состояние, возбуждаемое волной просвеления, может возникнуть при благориятном соотношении параметров, характеризующих кинетику просветления и релаксашию возбужденных состояний. Инвертированное состояние может быть создано как в конденсированном состоянии, например, в растворах органических и металлоорганических соединений, так и в разреженном газе, в частности, в астрофизических условиях. 0тметим, что для создания инвертированного состояния в космических условиях в микроволновой области, возбуждаемого оптической накачкой, то есть космического квантового усилителя (мазера), не требуется полного насьщения поглощения на частотах оптической накачки. Иначе говоря, прохождение волны просветления на частотах оптической накачки может быть достаточным условием для возбуждения космического мазера, но не является необходимьм. Упомянугый импульсный космический мазер, как и волны просветления, может возбуждаться различными нестационарными астрофизическими явлениями, в частности, периодическими и непериодическими вспышками звезд. Действие космического мазера может быть обнаружено, если нестационарные астроризические явления в оптической области спектра одновременно сопровождаются возрастанием интеисивности космического радиоизлучения в локальных областях спектра электромагнитных волн. Спектральные области, в которых может иметь место оптическая накачка и излучение космического мазера, рассмотрены Д. ВАРШАЛОВИЧЕМ [Писъма ЖЭТФ 4ь 180 (1966)], который также выказал предполохение о том, что когерентное усиление излучения - широко распространенное явление во вселенной. 